Research Article

\title{
Research on ARKFCM Algorithm Based on Membership Constraint and Bias Field Correction in Neonatal HIE Image Segmentation Method
}

\author{
Chao Huang $(\mathbb{D})$ and Jihua Wang \\ Shandong Normal University, Jinan, China \\ Correspondence should be addressed to Jihua Wang; jihuaaw@126.com
}

Received 1 August 2020; Revised 2 January 2021; Accepted 1 March 2021; Published 21 May 2021

Academic Editor: Suzanne M. Shontz

Copyright (c) 2021 Chao Huang and Jihua Wang. This is an open access article distributed under the Creative Commons Attribution License, which permits unrestricted use, distribution, and reproduction in any medium, provided the original work is properly cited.

\begin{abstract}
First, this paper presents the algorithm of adaptively regularized kernel-based fuzzy C-means based on membership constraint (GARKFCM). Under the idea of competitive learning based on penalizing opponents, a new membership constraint function penalty item is introduced for each sample point in the segmented image, so that the ARKFCM algorithm is no longer limited to the fuzzy index $m=2$. Secondly, the multiplicative intrinsic component optimization (MICO) is introduced into G-ARKFCM to obtain the GM-ARKFCM algorithm, which can correct the bias field when segmenting neonatal HIE images. Compared with other algorithms, the GM-ARKFCM algorithm has better segmentation quality and robustness. The GM-ARKFCM algorithm can more completely segment the neonatal ventricles and surrounding white matter and can retain more information of the original image.
\end{abstract}

\section{Introduction}

In the field of medical images and image analysis, image segmentation is one of the most important techniques. Image segmentation is to divide an image into multiple continuous nonoverlapping regions (such as gray, color, texture, and other information) with similar characteristics, so as to extract specific targets from a complex background.

Image segmentation technology [1] can be divided into threshold segmentation, clustering, modal-based, edge detection, and region growing methods. Some clustering-based image segmentation methods are proposed. Among them, the most popular method is the fuzzy C-means (FCM) clustering algorithm. Because it can retain more original image information than the hard clustering algorithm, it is also one of the most widely used fuzzy clustering algorithms.

The FCM algorithm was proposed and generalized by Bezdek [2]. The fuzzy C-means algorithm also has obvious shortcomings. It is sensitive to noise and does not consider the relationship between adjacent pixels in the image during the segmentation process. FCM algorithm uses the
Euclidean distance formula with poor robustness as a measure of pixels and cluster centers. In response to the above problems, related scholars have carried out a series of studies and improvements. Aiming at the fact that the FCM algorithm does not consider the problem of local information, Ahmed et al. [3] proposed the bias-corrected fuzzy C-means (BCFCM) algorithm, also known as the FCM_S algorithm. Since local information is introduced into the cost function, the accuracy of clustering is improved. However, each iteration calculation must consider every pixel in the neighborhood, which increases the running time. Chen and Zhang [4] proposed the FCM_S1 and FCM_S2 algorithms based on the FCM_S algorithm. Calculating the mean or median value of the neighborhood of the center pixel is equivalent to filtering and preprocessing the image, which greatly reduces the running time and improves the algorithm segmentation precision. At the same time, the parameters of the algorithm to weigh neighborhood information need to be set in advance. To this end, Krinidis and Chatzis [5] proposed a fuzzy local information C-means (FLICM) algorithm. The size of the control parameters is 
determined adaptively by introducing a fuzzy factor. However, it increases the complexity of the algorithm. In addition, fuzzy factors cannot measure heterogeneity. Aiming at the random selection of FCM algorithm clustering centers, Li et al. [6] proposed an improved gray wolf optimization algorithm with differential evolution (DEGWO) combined with fuzzy C-means to find the optimal initial center. Reddy and Das [7] proposed an image segmentation algorithm based on adaptive particle swarm optimization (APSO) and fuzzy C-means clustering. APSOF algorithm is better than FCM in correctly identifying the optimal clustering center, so that image pixels can be accurately classified.

In view of the FCM algorithm's sensitivity to noise due to the use of Euclidean distance, Zhang and Chen [8] proposed a kernel fuzzy C-means (KFCM) algorithm. By introducing a kernel function, the sample points in the original data space are nonlinearly mapped to the high-dimensional feature space and then clustered, which can effectively improve the clustering performance and improve the robustness to noise points and outliers. $\mathrm{Hu}$ and $\mathrm{Du}$ [9] proposed an improved kernel-based fuzzy C-means clustering algorithm, which uses the spatial neighborhood constraint characteristics of image pixels to introduce adaptive weighting coefficients into KFCM to automatically control the influence of neighborhood pixels on the central pixel. This method can accurately obtain the spatial information characteristics of the image and is robust to the realization of image segmentation. Gong et al. [10] enhanced the FLICM algorithm by using the kernel method to obtain the kernel weighted fuzzy local information C-means (KWFLICM) algorithm. Szilágyi [11] pointed out the theoretical problems of the FLICM algorithm and the KWFLICM algorithm: the algorithm does not have a minimum objective function and the objective function of the algorithm is theoretically problematic. To solve the above problems, Elazab et al. [12] proposed an adaptively regularized kernel-based fuzzy C-means (ARKFCM) algorithm. This method uses local heterogeneity information of the image, can adapt to local noise, can obtain clustering parameters in advance, and has less computational overhead. Xu et al. [13] proposed an MR image segmentation method (MPCFCM) with antinoise and bias field correction. The MPCFCM algorithm can better correct the bias field, eliminate noise, and obtain more detailed and accurate image segmentation results. The algorithm also constructs a new antinoise smoothing factor that suppresses the energy function, which makes the antinoise performance better and retains more image details. Kouhi et al. [14] proposed an FCM clustering algorithm with joint spatial constraints and membership matrix local information (FCMS-MLI) for brain MRI segmentation. The algorithm is more robust to noise and other artifacts.

The fuzzy index $m$ in the objective function of the FCM algorithm has an effect on the performance of the FCM algorithm. Bezdek [2] thinks that "the parameter $m$ controls the degree of sharing between fuzzy classes," but no strict proof is given. To realize the FCM algorithm, an appropriate value of $m$ must be selected, but how to choose an optimal $m$ still lacks theoretical guidance. Pal and Bezdek's experimental research
[15] on the effectiveness of clustering shows that the optimal selection interval for $m$ is $[1.5,2.5]$, and the median $m=2$ can be taken without special requirements. The choice of fuzzy index $m$ has an important impact on the clustering results [16], which can effectively improve the performance of the algorithm. Zhu et al. [17] proposed a generalized improved fuzzy partitioning fuzzy C-means (GIFP_FCM) algorithm, which solved the generalization problem of FCM algorithm fuzzy index $m$. By introducing the fuzzy degree coefficient, the GIFP-FCM algorithm can obtain the optimal solution no matter when the value of the weighting index $m$ is appropriate or when the value of $m$ is relatively large.

The existence of bias fields in MR images has always been a difficult problem in medical image segmentation. Solving the problem of bias fields in MR images during image segmentation can further improve the performance of the algorithm. This paper proposes an ARKFCM algorithm based on membership constraint and bias field correction. By introducing the fuzzy degree coefficient, the weighting index $m$ is made more flexible, so that the ARKFCM algorithm converges faster on the original basis, and the clustering quality and robustness of the algorithm are improved. Through the estimation and elimination of the bias field, the performance of the algorithm is further improved. Compared with other algorithms, this algorithm has higher segmentation accuracy.

Neonatal hypoxic-ischemic encephalopathy (HIE) is the main cause of neonatal death and neurological sequelae. MRI examination is increasingly widely used in the diagnosis of HIE because it does not require X-rays and has high image clarity [18]. The brain size of newborns is small, and the scanning time is much shorter than that of adults, resulting in low contrast-to-noise ratio, low signal-to-noise ratio, and low spatial resolution [19]; we use GM-ARKFCM algorithm to segment HIE images and compare them with other algorithms. The clustering effect is better. The GMARKFCM algorithm can segment the white matter of the neonatal ventricle and its surroundings when the value is appropriate, and the segmented image can retain more information about the original image.

\section{Related Work}

The FCM algorithm is an iterative algorithm based on the minimum quadratic criterion, in which clusters are represented by their respective centers. The idea is that the objects that are divided into the same cluster have the highest similarity and the smallest similarity between different clusters. The fuzzy C-means algorithm is an improvement of the ordinary C-means algorithm. It is hard for the ordinary C-means algorithm to divide the data, while FCM is more flexible for performing fuzzy division. The objective function of the fuzzy C-means clustering algorithm is as follows:

$$
J_{\mathrm{FCM}}=\sum_{i=1}^{N} \sum_{j=1}^{c} u_{i j}^{m}\left\|x_{i}-v_{j}\right\|^{2},
$$

where $N$ is the total number of pixels in the picture; $c$ is the number of cluster centers set; $u_{i j}$ is the membership between 
the $i$-th pixel and the $j$-th class; $m$ is the metric of fuzzy membership $u_{i j}$; and $\left\|x_{i}-x_{j}\right\|^{2}$ is the Euclidean distance between the $i$-th pixel and the cluster center $j$. The objective function satisfies the following conditions:

$$
\begin{aligned}
& \forall i \in[1, N], \\
& j \in[1, c], \\
& m \in(1, \infty), \\
& u_{i j} \in[0,1], \\
& \sum_{j=1}^{c} u_{i j}=1, \\
& 0 \leq \sum_{i=1}^{N} u_{i j} \leq N .
\end{aligned}
$$

The Lagrange operator method is used to calculate the minimum value of the objective function for formula (1). When the algorithm converges, the formulas of the membership a $u_{i j}$ and the center $v_{j}$ are obtained.

$$
\begin{aligned}
u_{i j} & =\frac{1}{\sum_{k=1}^{c}\left(\left\|x_{i}-v_{j}\right\|^{2} /\left\|x_{i}-v_{k}\right\|^{2}\right)^{1 /(m-1)}}, \\
v_{j} & =\frac{\sum_{i=1}^{N} u_{i j}^{m} x_{i}}{\sum_{i=1}^{N} u_{i j}^{m}} .
\end{aligned}
$$

ARKFCM algorithm is improved on the basis of FLICM algorithm and KWFLICM algorithm. The algorithm uses local variation coefficient (LVC) to measure the noise intensity of the image. When the noise is large, the heterogeneity of a pixel and its neighboring pixels is high, and the value of LVC will increase. The LVC expression is as follows:

$$
\operatorname{LVC}_{i}=\frac{\sum_{k \in N_{i}}\left(x_{k}-\bar{x}_{i}\right)^{2}}{N_{R} *\left(\bar{x}_{i}\right)^{2}}
$$

where $x_{k}$ is the gray value of the pixel in the neighborhood window $N_{i}$ of a pixel $i, N_{R}$ is the number of pixels in the neighborhood of $N_{i}$, and $\bar{x}_{i}$ is the mean value of the pixel gray in the neighborhood window. Applying LVC to the exponential function, we can get the weights in the neighborhood window from it. LVC is then projected into kernel space to make the weights regular. The results are as follows:

$$
\begin{gathered}
\zeta_{i}=\exp \left(\sum_{k \in N, i \neq k} \mathrm{LVC}_{k}\right), \\
\omega_{i}=\frac{\zeta_{i}}{\sum_{k \in N_{i}} \zeta_{k}} .
\end{gathered}
$$
by

The final weight assigned to each pixel can be calculated

$$
\phi_{i}= \begin{cases}2+\omega_{i}, & \bar{x}_{i}<x_{i}, \\ 2-\omega_{i}, & \bar{x}_{i}>x_{i}, \\ 0, & \bar{x}_{i}=x_{i} .\end{cases}
$$

In addition to the gray average or median filter representation of the image, $x$ can also be given by the weighted image $\xi$ :

$$
\xi_{i}=\frac{1}{2+\max \left(\phi_{i}\right)}\left(x_{i}+\frac{1+\max \left(\phi_{i}\right)}{N_{R}-1} \sum_{r \in N_{i}} x_{r}\right),
$$

where $x_{r}$ and $N_{i}$ are the gray value and neighborhood of pixel $i$, respectively. Through $\phi_{i}$, the neighborhood information can be adaptively weighed. It can be seen from the literature $[20,21]$ that the Euclidean distance in the expression can be replaced with the kernel induced distance. The formula replacement results are as follows:

$$
\left\|\phi\left(x_{i}\right)-\phi\left(v_{j}\right)\right\|^{2}=K\left(x_{i}, x_{i}\right)+K\left(v_{j}, v_{j}\right)-2 K\left(x_{i}, v_{j}\right) .
$$

The aforementioned kernel function $\mathrm{K}$ uses GRBF kernel, and the expression of kernel function $\mathrm{K}$ is as follows:

$$
K\left(x_{i}, v_{j}\right)=\exp \left(-\frac{\left\|x_{i}-v_{j}\right\|^{2}}{2 \sigma^{2}}\right),
$$

where $\sigma$ is the core width. It can be deduced from [10] that the distance change $\sigma$ based on pixels is

$$
\sigma=\left[\frac{\sum_{i=1}^{N}\left(d_{i}-\bar{d}\right)^{2}}{N-1}\right]^{1 / 2} .
$$

Using the GRBF core, equation (7) can be simplified to the following equation:

$$
\left\|\phi\left(x_{i}\right)-\phi\left(v_{j}\right)\right\|^{2}=2\left(1-K\left(x_{i}, v_{j}\right)\right) .
$$

The objective function of the ARKFCM algorithm is

$$
\begin{aligned}
J_{A R K F C M}= & 2\left[\sum_{i=1}^{N} \sum_{j=1}^{c} u_{i j}^{m}\left(1-K\left(x_{i}-v_{j}\right)\right)\right. \\
& \left.+\sum_{i=1}^{N} \sum_{j=1}^{c} \varphi_{i} u_{i j}^{m}\left(1-K\left(\bar{x}_{i}-v_{j}\right)\right)\right] .
\end{aligned}
$$

The Lagrange multiplier method is used to calculate the objective function to obtain the membership matrix and clustering center expression of the objective function:

$$
\begin{aligned}
u_{i j} & =\frac{\left(\left(1-K\left(x_{i}, v_{j}\right)\right)+\varphi_{i}\left(1-K\left(\bar{x}_{i}, v_{j}\right)\right)\right)^{-1 /(m-1)}}{\sum_{k=1}^{c}\left(1-K\left(x_{i}, v_{k}\right)+\varphi_{i}\left(1-K\left(\bar{x}_{i}, v_{k}\right)\right)\right)^{-1 /(m-1)}}, \\
v_{j} & =\frac{\sum_{i=1}^{N} u_{i j}^{m}\left(K\left(x_{i}, v_{j}\right) x_{i}+\varphi_{i} K\left(\bar{x}_{i}, v_{j}\right) \bar{x}_{i}\right)}{\sum_{i=1}^{N} u_{i j}^{m}\left(K\left(x_{i}, v_{j}\right)+\varphi_{i} K\left(\bar{x}_{i}, v_{j}\right)\right)} .
\end{aligned}
$$


The fuzzy index $m$ selected by the ARKFCM algorithm during calculation is 2 . However, the optimal value of the fuzzy index $m$ for different samples is not fixed. Selecting the same fuzzy index $m$ for different samples is a limitation on the performance of the algorithm. If you can break the limit of the fuzzy index $m$ value, you can improve the performance of the algorithm. The ARKFCM algorithm is optimized by improving the generalization problem of the fuzzy index $m$. From [13], we can see an improved GIFP_FCM algorithm based on the membership constraint function of the IFPFCM [22] algorithm. The algorithm is based on competitive learning $[23,24]$. There are two competitive learning rules: winner-take-all principle and winner can get more principles. For each data sample, it can make its corresponding clustering center have a higher degree of membership, and other clustering centers have a lower degree of membership. It can be seen from the literature [25] that in the clustering calculation, each data sample is attractive to all clustering centers. Not only does it generate a strong attraction that makes the cluster center closest to it closer to it during iteration, but it also produces attraction of different sizes for other cluster centers. Therefore, when a class center is attracted by similar data samples and close, it will also be attracted by other data samples, which is not conducive to the clustering of the algorithm.

The degree of membership can be understood as the magnitude of the attraction of the data sample to the cluster center. The greater the degree of membership, the greater the attraction to the center of the cluster. In this way, it is less affected by other clustering centers during clustering, which improves the convergence speed of the algorithm. The algorithm membership constraint function is introduced into the ARKFCM algorithm to obtain the GM-ARKFCM algorithm based on membership constraint. First, introduce the penalty term of the membership constraint function:

$$
\begin{aligned}
f\left(u_{i j}\right)= & \sum_{i=1}^{N} a_{i} \sum_{j=1}^{c} u_{i j}^{m}\left(1-u_{i j}^{m-1}\right), \\
a_{i}= & \alpha \cdot \min \left\{\left(\left(2-2 K\left(x_{i}, v_{j}\right)\right)\right.\right. \\
& \left.\left.+\varphi_{i}\left(2-2 K\left(\bar{x}_{i}, v_{j}\right)\right)\right), \quad j \in\{1,2, L, c\}\right\},
\end{aligned}
$$

where $\alpha$ is the fuzzy degree coefficient, and the size of $\alpha$ controls the maximum attraction of the sample point to the nearest cluster center. The value range of $\alpha$ is [0.1). The greater the attraction, the faster the convergence speed of the cluster center. Similarly, for cluster centers that are far away from each other, the attractive distance decreases accordingly, which can ensure the robustness of the cluster. The attractiveness of the winner increases with the increase of $\alpha$. When the blur degree coefficient is 0 , the algorithm changes back to the algorithm before improvement. We introduce the penalty term of the algorithm membership constraint function into the ARKFCM algorithm to form a new objective function:

$$
\begin{aligned}
J_{G-\mathrm{ARKFCM}}= & 2\left[\sum_{i=1}^{N} \sum_{j=1}^{c} u_{i j}^{m}\left(1-K\left(x_{i}, v_{j}\right)\right)\right. \\
& \left.+\sum_{i=1}^{N} \sum_{j=1}^{c} \phi_{i} u_{i j}^{m}\left(1-K\left(\bar{x}_{i}, v_{j}\right)\right)\right]+\sum_{i=1}^{N} a_{i} \sum_{j=1}^{c} u_{i j}^{m}\left(1-u_{i j}^{m-1}\right) .
\end{aligned}
$$

Using the Lagrange multiplier method, the membership matrix and clustering center expression of the objective function are obtained as follows:

$$
\begin{aligned}
u_{i j} & =\frac{\left(\left(2-2 K\left(x_{i}, v_{j}\right)\right)+\varphi_{i}\left(2-2 K\left(\bar{x}_{i}, v_{j}\right)\right)-a_{i}\right)^{-1 /(m-1)}}{\sum_{k=1}^{c}\left(2-2 K\left(x_{i}, v_{k}\right)+\varphi_{i}\left(2-2 K\left(\bar{x}_{i}, v_{k}\right)\right)-a_{i}\right)^{-1 /(m-1)}} \\
v_{j} & =\frac{\sum_{i=1}^{N} u_{i j}^{m}\left(K\left(x_{i}, v_{j}\right) x_{i}+\varphi_{i} K\left(\bar{x}_{i}, v_{j}\right) \bar{x}_{i}\right)}{\sum_{i=1}^{N} u_{i j}^{m}\left(K\left(x_{i}, v_{j}\right)+\varphi_{i} K\left(\bar{x}_{i}, v_{j}\right)\right)}
\end{aligned}
$$

During the acquisition of MR images, due to the uneven magnetic field, a flat, low-frequency bad signal is generated. The bias field will blur the image, lose part of the effective information, and cause the gray value of the same tissue to be different. Before segmentation or at the same time, the bias of the MR image can be eliminated, which can further improve the segmentation result [26]. The bias field correction multiplicative intrinsic component optimization (MICO) can be used for bias field estimation and correction. The grayscale modal of MICO for MR image is as follows:

$$
I(x)=b(x) J(x)+n(x)
$$

where $I(x)$ is the original image, $b(x)$ is the grayscale bias field, $J(x)$ is the real image, and $n(x)$ is the noise $(n(x)$ is not considered when removing the bias field from the image). By omitting the operation of $n(x)$ in the above formula, the formula can be changed to

$$
J(x)=\frac{I(x)}{b(x)} .
$$

By removing $b(x)$, a grayscale bias field, hypothetical real image $J(x)$ can be obtained. The gray bias field $b(x)$ can be expressed as follows:

$$
b(x)=w^{T} G(x) .
$$

Use column vector $w=\left(w_{1}, \ldots, w_{M}\right)^{T}$ to represent the coefficient $w_{1}, \ldots, w_{M}$; the basic function $g_{1}(x), \ldots, g_{M}(x)$ is represented by a column vector energy function $G(x)=\left(g_{1}(x), \ldots, g_{M}(x)\right)^{T}$. For real images $J(x)$, the similar clustering method is expressed as

$$
J(x)=\sum_{i=1}^{N} c_{i} u_{i}(x) .
$$

For a certain pixel $u_{i}$ of the image, the values are only 0 and 1 . The energy formula of the algorithm is 


$$
F(b, J)=F(u, c, w)=\int_{\Omega}\left|I(x)-w^{T} G(x) \sum_{i=1}^{N} c_{i} u_{i}(x)\right|^{2} \mathrm{~d} x,
$$

where $u_{i}$ is the membership function of area $\Omega_{i}$, when $x \notin \Omega_{i}$ has $u_{i}(x)=0$. When $x \in \Omega_{i}$, the following can be obtained:

$$
\sum_{i=1}^{N} c_{i} u_{i}(x)=c_{i} \text {. }
$$

The energy formula can be reduced to

$$
F(b, J)=F(u, c, w)=\int_{\Omega} \sum_{i=1}^{N}\left|I(x)-w^{T} G(x) c_{i}\right|^{2} u_{i}(x) \mathrm{d} x .
$$

Introduce fuzzer $q$ (the value of fuzzer $q$ is greater than or equal to 1) so that it can become soft segmentation, and the energy formula becomes

$$
F(b, J)=F(u, c, w)=\int_{\Omega} \sum_{i=1}^{N}\left|I(x)-w^{T} G(x) c_{i}\right|^{2} u_{i}^{q}(x) \mathrm{d} x .
$$

The minimization of energy can be achieved by fixing other values and fixing $c$ and $u$ to obtain

$$
\begin{gathered}
w=\left(\int_{\Omega} G(x) G^{T}(x)\left(\sum_{i=1}^{N} c_{i}^{2} u_{i}^{q}(x)\right) \mathrm{d} x\right)^{-1} \\
\cdot \int_{\Omega} G(x) I(x)\left(\sum_{i=1}^{N} c_{i} u_{i}^{q}(x)\right) \mathrm{d} x .
\end{gathered}
$$

The bias field can be estimated by the above formula and $b(x)=w^{T} G(x)$ to obtain the bias field $b(x)$.

The MR image obtained by MICO is regarded as the image obtained by multiplying the grayscale biased field in an ideal state. Select neonatal HIE images for image bias field correction, and the results are as follows.

It can be seen from Figure 1 that the HIE image of the neonate after the bias field is removed cannot be seen with the naked eye, so it can be judged by drawing the gray histogram of the image. It can be seen from the image grayscale histogram that the grayscale value of the image is not closely distributed before the image processing, and the grayscale value of the image is more uniform after the bias field is corrected.

Aiming at the image fidelity problem of neonatal HIE images after correcting the bias field, since the undistorted image cannot be obtained as a reference image, the reference-free image quality evaluation NIQE algorithm proposed in [27] is used to evaluate the objective quality of images before and after segmentation. The calculation results of the original image of the NIQE algorithm and the real image after removing the bias field are as follows.

The lower the value of NIQE algorithm, the higher the objective evaluation of the image. From the score of the original image and the score of the image after removing the bias field, it is known from Table 1 that the image quality after removing the bias field is not reduced, but the image quality is improved slightly. It reflects from the side that most of the original image information has been effectively retained.

Combined with MICO, a GM-ARKFCM algorithm that can estimate and correct the bias field of segmented images is proposed. The calculation steps of the GM-ARKFCM algorithm are as follows:

(1) Input original image.

(2) Update the bias field by formula (27) and formula (21).

(3) Use formula (20) to get the real image $J(x)$.

(4) Initialize the number of clusters $c$ and set the threshold $\varepsilon$, fuzzy index $m$, fuzzy degree $\alpha$, iteration number $T$, cluster center $v$, and membership degree $u^{(0)}$.

(5) Use formula (7) to calculate adaptive regularization parameters $\phi_{i}$.

(6) Calculate $\bar{x}_{i}$.

(7) Substitute $u^{(T)}$ into formula (18) to calculate $v_{j}^{(T)}$.

(8) Bring formula (17) to calculate $u^{(T+1)}$.

(9) If the value of $\left\|u^{(T+1)}-u^{(T)}\right\|$ is less than the set threshold $\varepsilon$ or the number of iterations $T$ exceeds the originally set maximum number of iterations, the algorithm is terminated after the above conditions are met. Otherwise, update the number of iterations and return to step (7).

\section{Experimental Study of Synthetic Brain MR Images}

In order to verify the performance of the GM-ARKFCM algorithm, the segmentation experiment of the synthetic brain MR image is analyzed. The synthetic brain MR image comes from the simulated brain database (SBD) [28], which contains two kinds of simulated brain MR data and provides the ground truth image for reference, from which we select the normal simulated brain MR data. Under T1 modal and ICMB protocol, the 90th brain image with slice thickness of $1 \mathrm{~mm}$, noise level of 7\%, and gray level of $40 \%$ and the 80th brain image with slice thickness of $1 \mathrm{~mm}$, noise level of $9 \%$, and gray level of $20 \%$ were segmented. We select MICO algorithm [29], ARKFCM algorithm, and GM-ARKFCM algorithm with different fuzzy index $m$. The range of the fuzzy index is $[1.5,2.5]$. Get the segmented image of each algorithm, compare the segmented result with the ground truth image quantitatively, and the segmentation accuracy can be measured by Jaccard similarity (JS) [30]:

$$
\operatorname{JS}(A, B)=\frac{|A \cap B|}{|A \cup B|},
$$

where $A$ and $B$ represent the segmented image result and ground truth image. The higher the accuracy of the segmentation, the larger the JS. The experimental results are shown below. 


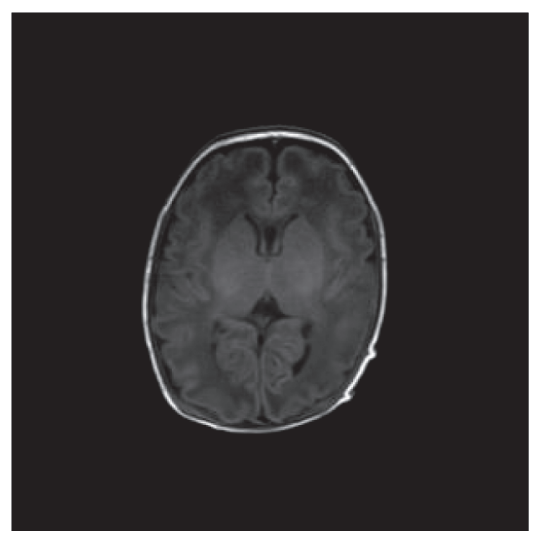

(a)

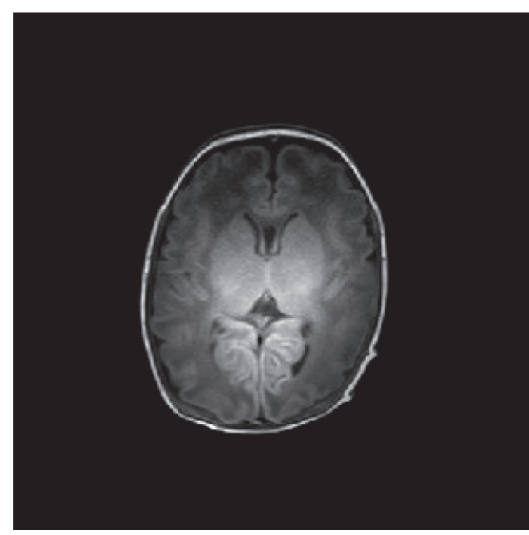

(c)

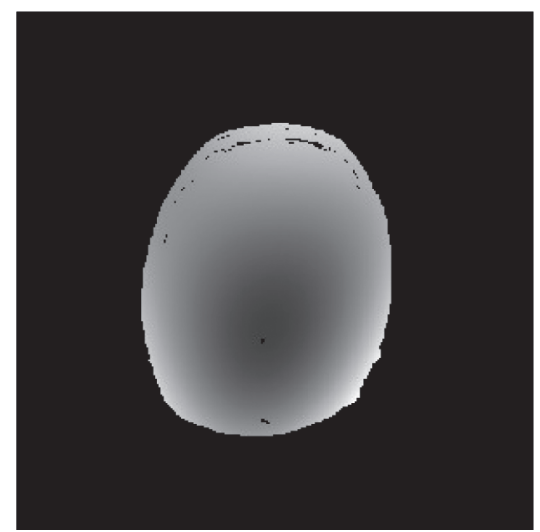

(b)

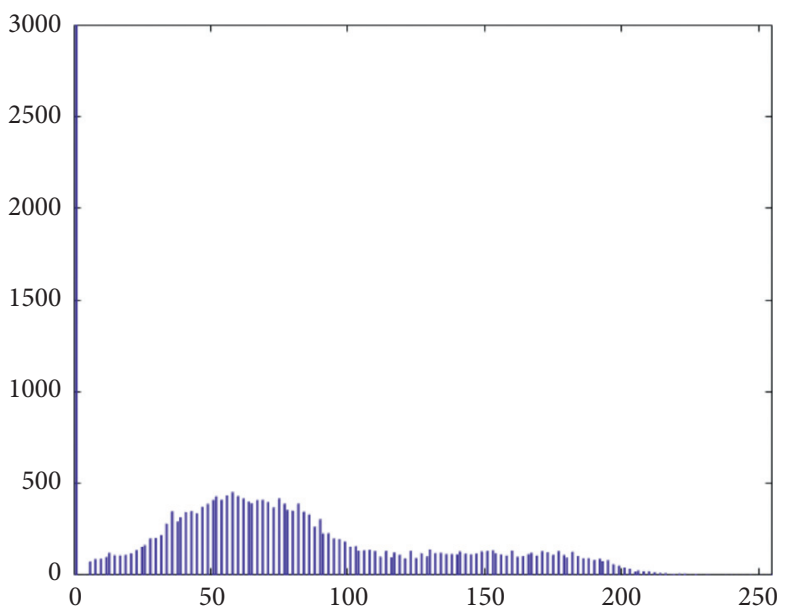

(d)

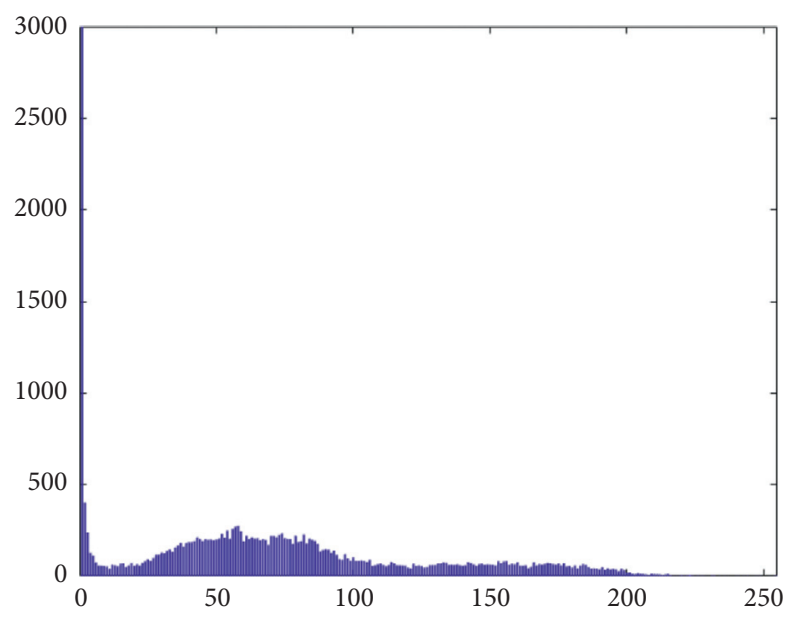

(e)

FIgURE 1: Correction of the bias field in the MR image of neonatal hypoxic-ischemic encephalopathy. (a) Original image. (b) Image bias field. (c) Real image. (d) Gray histogram of (a). (e) Gray histogram of (c).

TABLE 1: The quality of neonatal HIE images before and after removing the bias field.

\begin{tabular}{lcc}
\hline Image & Original image & Real image \\
\hline Quality & 6.1533 & 6.0384 \\
\hline
\end{tabular}

From Figures 2 and 3, we can see that the GM-ARKFCM algorithm has better segmentation effect and better robustness to noise. From Tables 2 and 3, the GM-ARKFCM algorithm has better segmentation results for images with noise and uneven grayscale, and the clustering performance is better than 


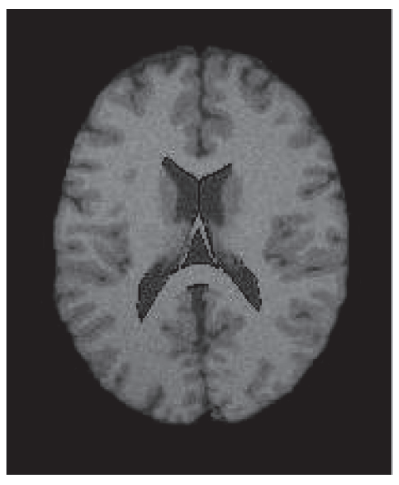

(a)

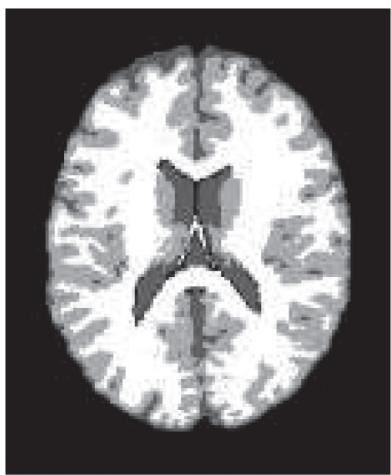

(e)

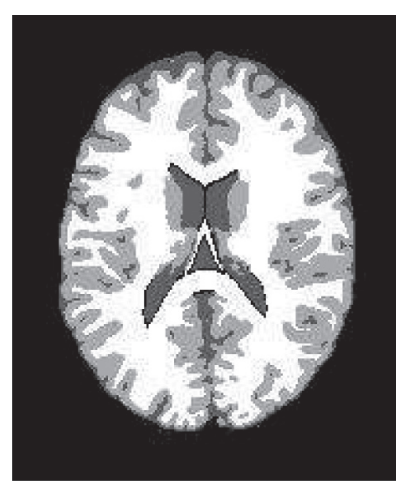

(b)

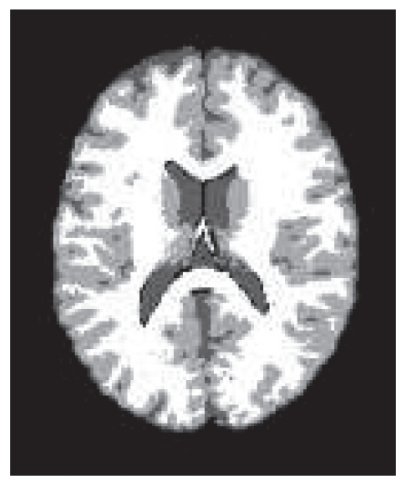

(f)

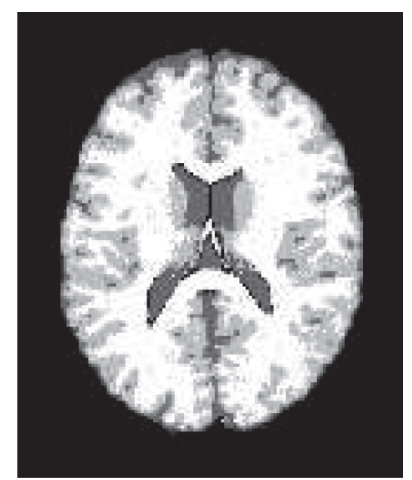

(c)

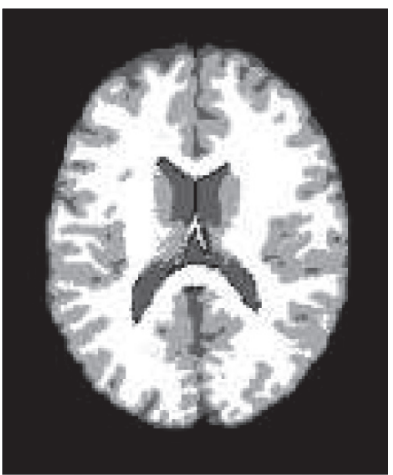

(g)

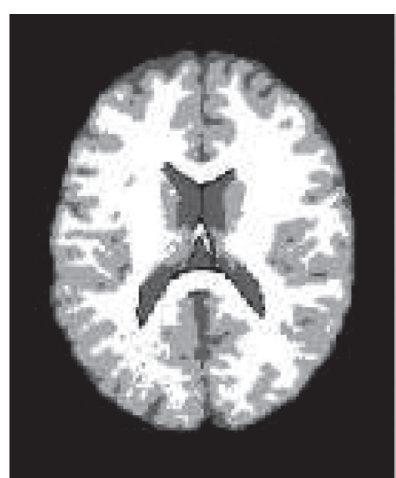

(d)

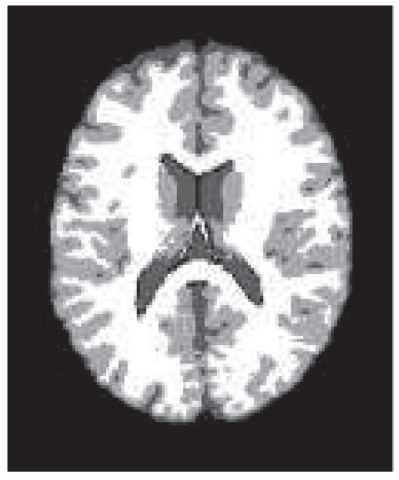

(h)

FIGURE 2: For the T1 modal, under the ICMB protocol, the slice thickness is $1 \mathrm{~mm}$, the noise level is 7\%, and the gray unevenness level is the 90th layer of brain image segmentation results. (a) Original image. (b) Ground truth. (c) MICO algorithm segmentation result. (d) ARKFCM algorithm segmentation result. (e) GM-ARKFCM algorithm segmentation result $(m=1.8, \alpha=0.99)$. (f) GM-ARKFCM algorithm segmentation result $(m=2.2, \alpha=0.9)$. (g) GM-ARKFCM algorithm segmentation result $(m=2.2, \alpha=0.99)$. (h) GM-ARKFCM algorithm segmentation result $(m=2.4, \alpha=0.99)$.

other algorithms. From the accuracy of the segmentation, the GM-ARKFCM algorithm can obtain more ideal results under different values of the fuzzy index $m$. When the value of fuzzy index $m$ is 2 , it does not represent the optimal solution of the algorithm. The diversity of the value of fuzzy index $m$ increases the flexibility of the algorithm and brings more possibilities to the algorithm. However, in terms of segmentation accuracy, there are still large gaps between segmentation results with different values of $m$. How to choose the appropriate fuzzy index $m$ still needs to increase the contrast test.

When the segmented MR image contains significant noise, compared to the results of the image segmentation by the MICO algorithm and the ARKFCM algorithm, the image segmented by the GM-ARKFCM algorithm obviously contains a smaller amount of noise, which proves that the GMARKFCM algorithm is very robust to noise.

When the value of the fuzzy index $m$ is fixed, the selection of the fuzzy degree coefficient determines the segmentation result. It can be seen from the table that when the value of $m$ is 2.2, the larger the value of the blur degree coefficient, the better the segmentation result. Therefore, when there is no requirement for the fuzzy degree coefficient, try to select the maximum value.

In summary, the GM-ARKFCM algorithm performs better than the ARKFCM algorithm when segmenting images, and it is robust against noisy images. GM-ARKFCM has more choices in the selection of fuzzy index $m$ and is no longer limited to the case where the value of fuzzy index $m$ is 2, making the algorithm more flexible.

\section{Segmentation of Neonatal HIE Images}

4.1. Image Segmentation. Select the neonatal HIE image using KWFLICM algorithm, FRFCM algorithm [31], and ARKFCM algorithm for segmentation. In order to obtain better segmentation results, the fuzzy index $m$ is set to be 2.0 and 2.2, and the fuzzy degree coefficient is set to be 0.99 . The experimental results of segmenting neonatal HIE images are as follows.

It can be seen from Figure 4 that due to the selection of neonatal HIE images with greater noise and lower spatial resolution, it is not ideal for the segmented image to retain the information of the original image. KWFLICM algorithm has strong segmentation ability, but the segmentation result of neonatal ventricular area is poor, and the general shape of the ventricular area cannot be segmented. The FRFCM algorithm cannot segment a complete ventricle image, and the segmentation retains less original image information and lacks robustness to noise. Compared with the FRFCM algorithm, the ARKFCM algorithm is greatly improved in 


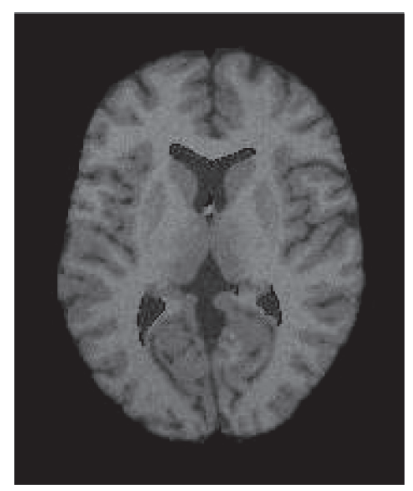

(a)

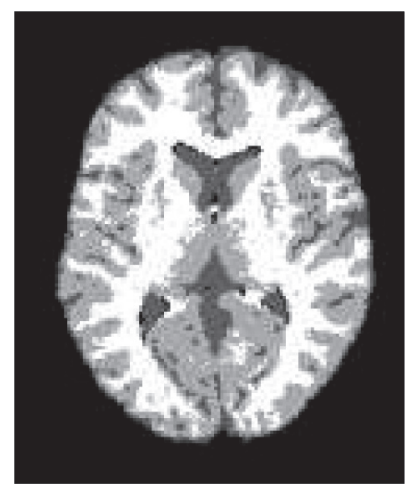

(e)

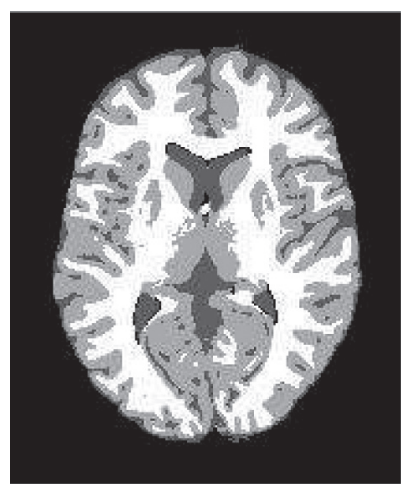

(b)

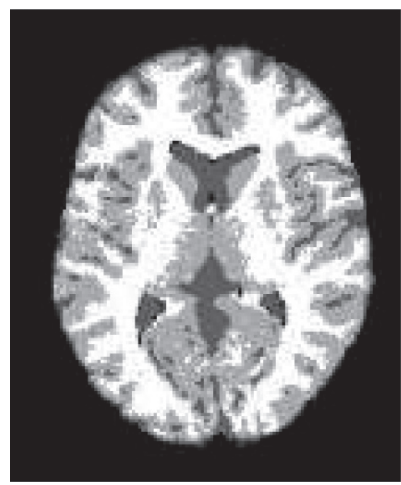

(f)

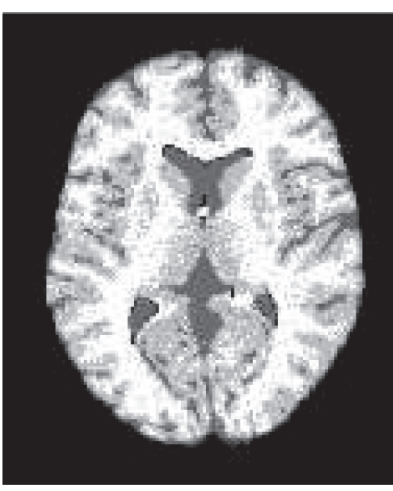

(c)

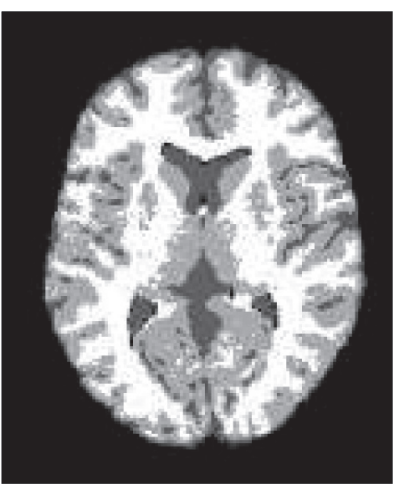

(g)

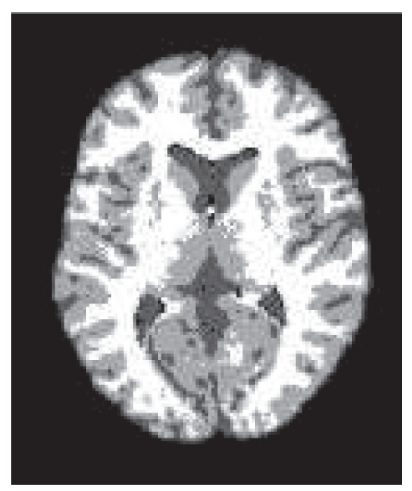

(d)

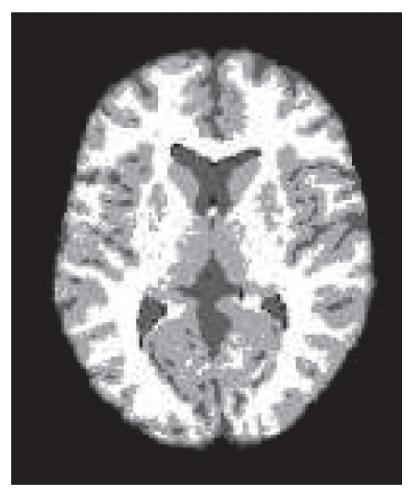

(h)

Figure 3: For T1 modal, under the ICMB protocol, the slice thickness is $1 \mathrm{~mm}$, the noise level is $9 \%$, and the gray unevenness level is the 80th layer of brain image segmentation results. (a) Original image. (b) Ground truth. (c) MICO algorithm segmentation result. (d) ARKFCM algorithm segmentation result. (e) GM-ARKFCM algorithm segmentation result $(m=1.8, \alpha=0.99)$. (f) GM-ARKFCM algorithm segmentation result $(m=2.0, \alpha=0.99)$. (g) GM-ARKFCM algorithm segmentation result $(m=2.2, \alpha=0.99)$. (h) GM-ARKFCM algorithm segmentation result $(m=2.4, \alpha=0.99)$.

TABLE 2: JS quantitative analysis result of the 90th layer brain image with a slice thickness of $1 \mathrm{~mm}$, a noise level of 7\%, and a gray unevenness level of $40 \%$ under the T1 modal and ICMB protocol.

\begin{tabular}{lcccccc}
\hline Algorithm & MICO & ARKFCM & $\begin{array}{c}\text { GM-ARKFCM } \\
(m=1.8, \alpha=0.99)\end{array}$ & $\begin{array}{c}\text { GM-ARKFCM } \\
(m=2.2, \alpha=0.9)\end{array}$ & $\begin{array}{c}\text { GM-ARKFCM } \\
(m=2.2, \alpha=0.99)\end{array}$ & $\begin{array}{c}\text { GM-ARKFCM } \\
(m=2.4, \alpha=0.99)\end{array}$ \\
\hline WM & 0.828 & 0.872 & 0.904 & 0.915 & 0.916 & 0.881 \\
GM & 0.786 & 0.832 & 0.872 & 0.880 & 0.897 & 0.875 \\
CSF & 0.811 & 0.828 & 0.891 & 0.892 & 0.898 & 0.873 \\
Average & 0.808 & 0.844 & 0.889 & 0.895 & 0.885 \\
\hline
\end{tabular}

TABLE 3: JS quantitative analysis result of the 80th layer of the brain image with a slice thickness of $1 \mathrm{~mm}$, a noise level of $9 \%$, and a gray unevenness level of $20 \%$ under the T1 modal and ICMB protocol.

\begin{tabular}{|c|c|c|c|c|c|c|}
\hline Algorithm & MICO & ARKFCM & $\begin{array}{c}\text { GM-ARKFCM }(m=1.8, \\
\alpha=0.99)\end{array}$ & $\begin{array}{c}\text { GM-ARKFCM }(m=2.0, \\
\alpha=0.99)\end{array}$ & $\begin{array}{c}\text { GM-ARKFCM }(m=2.2, \\
\alpha=0.99)\end{array}$ & $\begin{array}{c}\text { GM-ARKFCM }(m=2.4, \\
\alpha=0.99)\end{array}$ \\
\hline WM & 0.764 & 0.816 & 0.880 & 0.883 & 0.887 & 0.881 \\
\hline GM & 0.726 & 0.835 & 0.856 & 0.870 & 0.875 & 0.868 \\
\hline CSF & 0.814 & 0.823 & 0.838 & 0.844 & 0.839 & 0.841 \\
\hline Average & 0.768 & 0.824 & 0.858 & 0.865 & 0.867 & 0.863 \\
\hline
\end{tabular}

terms of robustness to noise and can roughly segment the shape of the ventricular region, but it cannot effectively segment other white matter and gray matter regions. Compared with the original image, the GM-ARKFCM algorithm can segment the image with more details. Compared with the ARKFCM algorithm, the WM and GM of other regions can also be roughly segmented, and it has a good segmentation effect and robustness to noise. 


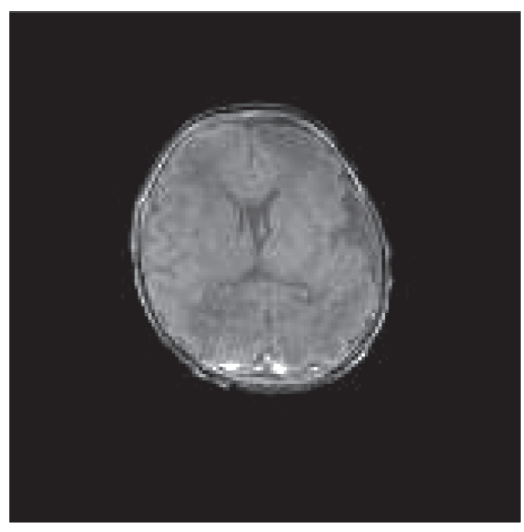

(a)

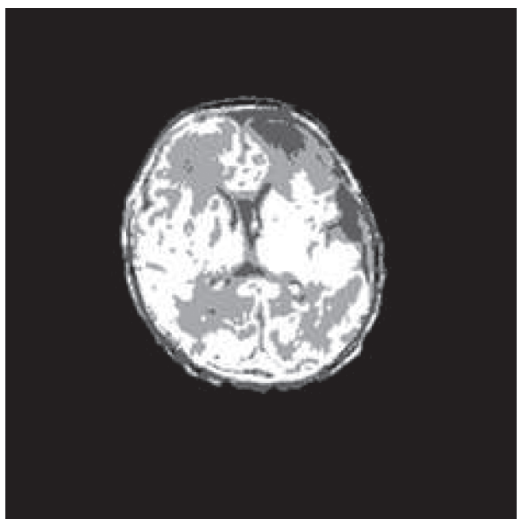

(d)

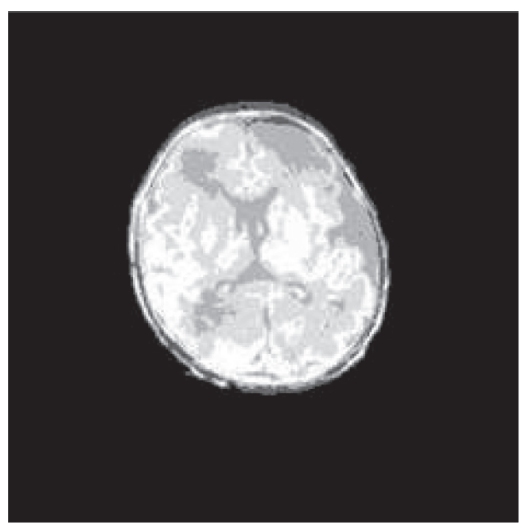

(b)

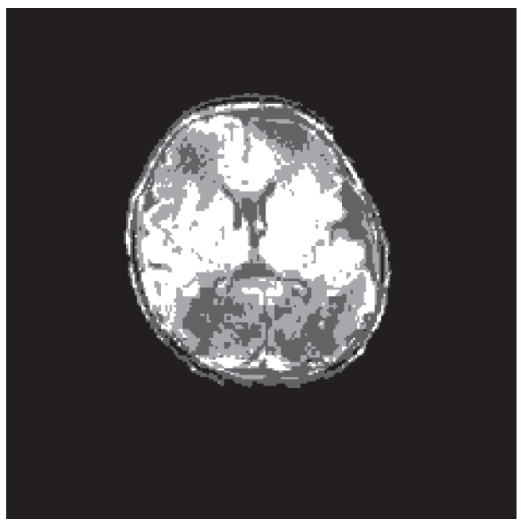

(e)

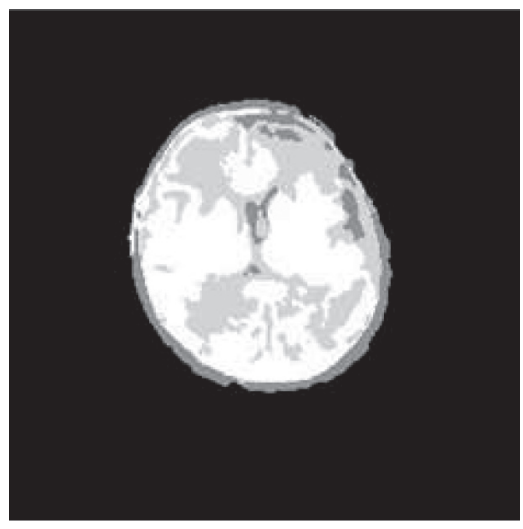

(c)

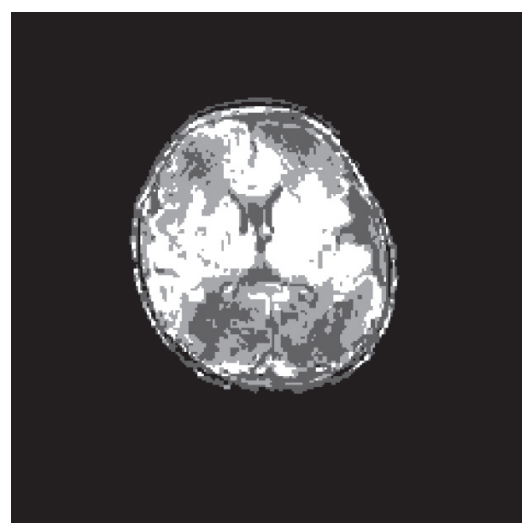

(f)

FIGURE 4: Segmentation results of neonatal HIE image. (a) Original image. (b) KWFLICM algorithm segmentation result. (c) FRFCM algorithm segmentation result. (d) ARKFCM algorithm segmentation result. (e) GM-ARKFCM algorithm segmentation result $(m=2.0$, $\alpha=0.99)$. (f) GM-ARKFCM algorithm segmentation result $(m=2.2, \alpha=0.99)$.

4.2. Segmentation Assessment. Supervised evaluation method can obtain accurate segmentation results, but there is no standard image as a reference for neonatal HIE image, so accurate results cannot be used to prove the actual effect of segmentation. Although unsupervised evaluation methods do not need standard images as a reference, they are all driven by the data of evaluating image features, which is difficult to be applied to medical image analysis. Therefore, the shape of ventricle area can only be segmented through threshold segmentation by analyzing the gray value range of the original image.

From the comparison between the segmented image and the original image, the ARKFCM algorithm and the GMARKFCM algorithm can segment the general shape of the ventricle area. We collect the ventricular ROI area of the original image and the segmented image and perform threshold segmentation on the ROI area. According to the gray level of the image, the approximate shape of the ventricle area is segmented. The segmentation results are as follows.

It can be seen from the shape of the ROI region after threshold segmentation in Figure 5 that the segmented shape of the GM-ARKFCM algorithm is closer to the original image. Calculating the approximate accuracy of the ROI area from Table 4, it can be seen that the ARKFCM algorithm can roughly determine the general shape of the neonatal HIE image, and less information about the ventricle can be obtained. Compared with the ARKFCM algorithm, the accuracy of the GM-ARKFCM algorithm has been greatly improved. It can segment the general shape of the neonatal HIE image, which has important reference significance.

Combined with the above image segmentation information, due to the fact that the original image is affected by various factors such as the neonatal brain volume and short scan time in actual operation, there are phenomena such as low contrast of the image, a lot of noise, and low spatial resolution. Other algorithms have poor noise robustness, and it is difficult to segment the shape of the ventricle and the surrounding WM and GM. The result of segmentation of the original image by the ARKFCM algorithm is also not satisfactory. It cannot segment a complete ventricle, and it cannot achieve accurate segmentation for some areas with loud noises. The ARKFCM algorithm can roughly segment the ventricles and some areas of neonatal hypoxic-ischemic encephalopathy, which is difficult to use as a reference in practical applications. It can be seen from the figure that the GM-ARKFCM algorithm can segment the shape of the ventricle region relatively complete when obtaining a relatively suitable value and segment the WM and GM shapes around the ventricle region more accurately. The 


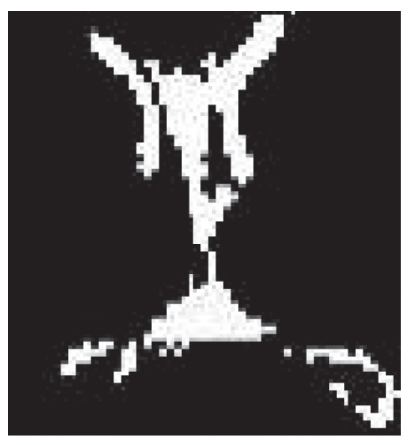

(a)

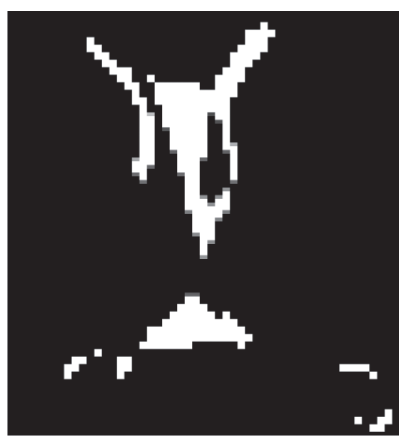

(b)

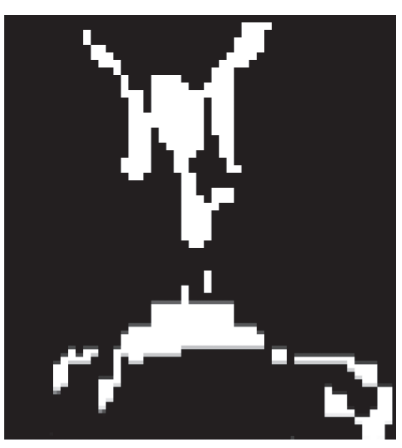

(c)

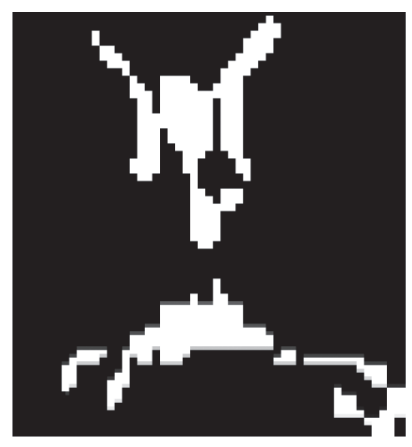

(d)

FIGURE 5: Threshold segmentation results of ROI region of original image and segmented image. (a) Original image. (b) ARKFCM algorithm segmentation result. (c) GM-ARKFCM algorithm segmentation result $(m=2.0, \alpha=0.99)$. (d) GM-ARKFCM algorithm segmentation result $(m=2.2, \alpha=0.99)$.

TABLE 4: JS quantitative analysis of the approximate accuracy of ARKFCM algorithm and GM-ARKFCM algorithm for ventricular segmentation.

\begin{tabular}{lccc}
\hline Algorithm & ARKFCM & GM-ARKFCM $(m=2.0, \alpha=0.99)$ & GM-ARKFCM $(m=2.2, \alpha=0.99)$ \\
\hline Accuracy & 0.502 & 0.584 & 0.604 \\
\hline
\end{tabular}

GM-ARKFCM algorithm also divides the noisy area into a general shape, which reflects that the robustness of the GMARKFCM algorithm is better than that of the ARKFCM algorithm.

\section{Conclusion}

The fuzzy C-means clustering algorithm of the adaptive kernel function constrained by the membership degree makes the fuzzy index $m$ of the ARKFCM algorithm no longer single, and the diversity of fuzzy indexes improves the performance of the algorithm. We optimize the image bias field by combining the multiplicative intrinsic component optimization to further improve the algorithm performance. Experiments show that the GM-ARKFCM algorithm is superior to the ARKFCM algorithm in clustering quality and robustness. The effect of the GM-ARKFCM algorithm on MR image segmentation of real neonatal hypoxic-ischemic encephalopathy shows that the algorithm can segment relatively complete neonatal ventricle and surrounding proteins. The algorithm can be used as a potential tool for segmenting neonatal hypoxic-ischemic encephalopathy. The value of the fuzzy index $m$ also needs specific research. From this article, it is impossible to determine whether the value of the fuzzy index $m$ is the optimal value, and the performance of the algorithm may be further improved. This will be the future research direction of this paper.

\section{Data Availability}

The data used to support the findings of this study are available from the corresponding author upon request.

\section{Conflicts of Interest}

The authors declare that they have no conflicts of interest.

\section{References}

[1] D. L. Pham, C. Xu, and J. L. Prince, "Current methods in medical image segmentation," Annual Review of Biomedical Engineering, vol. 2, no. 1, pp. 315-337, 2000.

[2] J. C. Bezdek, "Selected applications in classifier design," Pattern Recognition with Fuzzy Objective Function Algorithms, vol. 22, no. 1171, pp. 203-239, 1981.

[3] M. N. Ahmed, S. M. Yamany, N. Mohamed, A. A. Farag, and T. Moriarty, "A modified fuzzy c-means algorithm for bias field estimation and segmentation of MRI data," IEEE Transactions on Medical Imaging, vol. 21, no. 3, pp. 193-199, 2002.

[4] S. Chen and D. Zhang, "Robust image segmentation using FCM with spatial constraints based on new kernel-induced distance measure," IEEE Transactions on Systems, Man and Cybernetics, Part B (Cybernetics), vol. 34, no. 4, p. 1907, 2004.

[5] S. Krinidis and V. Chatzis, "A robust fuzzy local information C-means clustering algorithm," IEEE Transactions on Image Processing, vol. 19, no. 5, pp. 1328-1337, 2010.

[6] M. Q. Li, L. P. Xu, N. Xu, T. Huang, and B. Yan, "SAR image segmentation based on improved grey wolf optimization algorithm and fuzzy C-means," Mathematical Problems in Engineering, vol. 2018, no. 10, 11 pages, Article ID 4576015, 2018.

[7] A. N. Reddy and R. Das, "Color image segmentation using adaptive particle swarm optimization and fuzzy C-means," 2020, https://arxiv.org/abs/2004.08547.

[8] D.-Q. Zhang and S.-C. Chen, "A novel kernelized fuzzy C-means algorithm with application in medical image segmentation," Artificial Intelligence in Medicine, vol. 32, no. 1, pp. 37-50, 2004.

[9] G. Hu and Z. Du, "Adaptive kernel-based fuzzy C-means clustering with spatial constraints for image segmentation[J]," International Journal of Pattern Recognition and Artificial Intelligence, vol. 33, no. 1, pp. 1954003.1-1954003.15, 2019.

[10] M. Gong, Y. Liang, J. Shi, W. Ma, and J. Ma, "Fuzzy C-means clustering with local information and kernel metric for image 
segmentation," IEEE Transactions on Image Processing, vol. 22, no. 2, p. 573, 2013.

[11] G. L. Szil, Lessons to Learn from a Mistaken optimization, pp. 29-35, Elsevier Science Inc., London, UK, 2014.

[12] A. Elazab, C. Wang, F. Jia et al., "Segmentation of brain tissues from magnetic resonance images using adaptively regularized kernel-based fuzzy C-means clustering," Computational and Mathematical Methods in Medicine, vol. 2015, no. 5, pp. 485-495, 2015.

[13] H. Xu, C. Ye, F. Zhang et al., "A medical image segmentation method with anti-noise and bias-field correction[J]," IEEE Access, no. 99, p. 1, 2020.

[14] A. Kouhi, H. Seyedarabi, and A. Aghagolzadeh, "Robust FCM clustering algorithm with combined spatial constraint and membership matrix local information for brain MRI segmentation," Expert Systems with Application, vol. 146, no. May, pp. 113159.1-113159.16, 2020.

[15] N. R. Pal and J. C. Bezdek, "On cluster validity for the fuzzy C-means modal," IEEE Press, vol. 3, no. 3, pp. 370-379, 1995.

[16] G. Xinbo, P. Jihong, and X. Weixin, "A study of weighting exponent in a fuzzy -means algorithm," Acta Electronica Sinica, vol. 28, no. 4, pp. 80-83, 2000.

[17] L. Zhu, F. L. Chung, and S. Wang, "Generalized fuzzy C-means clustering algorithm with improved fuzzy partitions," IEEE Transactions on Cybernetics, vol. 39, no. 3, pp. 578-591, 2009.

[18] L. Liu, C.-x. Zheng, S.-f. Peng et al., "Evaluation of urinary S100B protein level and lactate/creatinine ratio for early diagnosis and prognostic prediction of neonatal hypoxicischemic encephalopathy," Neonatology, vol. 97, no. 1, pp. 41-44, 2010.

[19] S. Mukherjee, I. Cheng, S. Miller, T. Guo, V. Chau, and A. Basu, "A fast segmentation-free fully automated approach to white matter injury detection in preterm infants," Medical and Biological Engineering and Computing, vol. 57, no. 1, pp. 71-87, 2019.

[20] T. Hofmann, B. Schölkopf, and A. J. Smola, "Kernel methods in machine learning," The Annals of Statistics, vol. 36, no. 3, pp. 1171-1220, 2008.

[21] K.-R. Muller, S. Mika, G. Ratsch, K. Tsuda, and B. Scholkopf, "An introduction to kernel-based learning algorithms," IEEE Transactions on Neural Networks, vol. 12, no. 2, pp. 181-201, 2001.

[22] F. Hoppner and F. Klawonn, "Improved fuzzy partitions for fuzzyregression modals," Journal of Approximate Reasoning, vol. 32, no. 2, pp. 85-102, 2003.

[23] Y. J. Zhang and Z. Q. Liu, "Self-splitting competitive learning: a new on-line clustering paradigm," IEEE Trans on Neural Network, vol. 13, no. Z, pp. 369-380, 2002.

[24] S. H. Wu, A. W. Liew, Y. Hong et al., "Cluster analysis of gene expression data based on self-splitting and merging competitive learning," IEEE Transaction on Information Technology in Biomedicine, vol. 8, no. 1, pp. 5-15, 2004.

[25] L. Xu, A. Krzyak, and E. Oja, "Rival penalized competitive learning for clustering analysis, RBF net and curve detection," IEEE Transaction on Neural Network, vol. 4, no. 4, pp. 636-649, 1993.

[26] L. Chunming, J. C. Gore, and C. Davatzikos, "Multiplicative intrinsic component optimization (MICO) for MRI bias field estimation and tissue segmentation," Magnetic Resonance Imaging, vol. 32, no. 7, pp. 913-923, 2014.

[27] C. A. Cocosco, V. Kollokian, R. K.-S. Kwan, and A. C. Evans, BrainWeb: Online Interface to a 3D MRI Simulated Brain Database, http://www.bic.mni.mcgill.ca/brainweb/, 2011.
[28] C. Li, C. Xu, A. W. Anderson et al., "MRI tissue classification and bias field estimation based on coherent local intensity clustering: a unified energy minimization framework," Information Processing in Medical Imaging, vol. 21, pp. 288-299, 2009.

[29] C. Feng, D. Zhao, and M. Huang, "Image segmentation using CUDA accelerated non-local means denoising and bias correction embedded fuzzy c-means (BCEFCM)," Signal Processing, vol. 122, pp. 164-189, 2016.

[30] T. Lei, X. Jia, Y. Zhang et al., "Significantly fast and robust fuzzy C-means clustering algorithm based on morphological reconstruction and membership filtering," IEEE Transactions on Fuzzy Systems, p. 1, 2018.

[31] A. Mittal, R. Soundararajan, and A. C. Bovik, "Making a "completely blind" image quality analyzer," IEEE Signal Processing Letters, vol. 20, no. 3, pp. 209-212, 2013. 